\title{
Erratum: Spatiotemporal Evolution of an Ultrashort Pulse Pair in a Resonant Two-level Atomic Medium
}

\author{
[J. Korean Phys. Soc. 60, 1270 (2012)] \\ Yueping NiU* and Shangqing GoNG \\ Department of Physics, East China University of Science and Technology, Shanghai 200237, China \\ $\mathrm{Ni}$ CUI \\ Max Planck Institute for Nuclear Physics, Heidelberg, Germany
}

DOI: $10.3938 /$ jkps.62.1710

In our previous paper, the contribution of the third author, Ni Cui, to the paper was mainly made when she studied in Chinese Academy of Sciences. The address of the Max Planck Institute was used unsuitably and without permission. Now her affiliation is corrected to "State Key Laboratory of High Field Laser Physics, Shanghai Institute of Optics and Fine Mechanics, Chinese Academy of Sciences, Shanghai 201800, China". 